\title{
INEXACT NEWTON'S METHOD FOR GENERALIZED OPERATOR EQUATIONS IN BANACH SPACES
}

\author{
Vipin Kumar Singh \\ Department of Science and Humanities \\ Government Polytechnic Korea \\ Chhattishgarh, India-497335 \\ vipinkumarsingh666@gmail.com
}

Received: 11 April 2018; Accepted: 25 February 2019

\begin{abstract}
In the present paper, we introduce a new inexact Newton-like algorithm for solving the generalized operator equations containing non differentiable operators in Banach space setting and discuss its semilocal convergence analysis under the weak Lipschitz condition with larger convergence domain and tighter error bounds. The main result of this paper is the significant improvement over the Newton's method as well as the inexact Newton method.
\end{abstract}

MSC 2010: 49M15, 65J15

Keywords: nonlinear operator equation, Fréchet derivative, Newton's method, inexact Newton method

\section{Introduction}

Let $X, Y$ be two Banach spaces and $D$ a nonempty open convex subset of $X$ let $F: D \rightarrow Y$ be an operator. Solving a nonlinear operator equation $F(x)=0$ is a basic and very important problem in computational and applied mathematics. A powerful technique for solving such a problem is the Newton's method defined by

$$
w_{n+1}=w_{n}-F_{w_{n}}^{\prime-1} F\left(w_{n}\right), \quad n \in \mathbb{N}_{0},
$$

where $w_{0} \in D$ is the initial point and $\mathbb{N}_{0}=\mathbb{N} \cup\{0\}$. The semilocal convergence analysis of method was first studied by Kantorovich $[1,2]$. Kantorovich's result provides a simple and transparent convergence criterion for the nonlinear operator with bounded second Fréchet derivative $F^{\prime \prime}$. There are two disadvantages of Newton's method (1). The first step is to evaluate the Fréchet derivative of involved operator and the second is to find exact solution of the following Newton equation:

$$
F_{w_{n}}^{\prime}\left(w_{n+1}-w_{n}\right)=-F\left(w_{n}\right), n \in \mathbb{N}_{0}
$$

at each iteration. In many applications, when $F_{w_{n}}^{\prime}$ is too large and dense, it may not be feasible to solve equation (2) exactly. To avoid the first disadvantage of Newton's 
method, a number of Newton-like methods have been developed in literature. To overcome the second disadvantage of Newton's method, linear iterative methods have been extensively developed to find an approximate solution of Newton equation (2) (see [3-8]). These methods have been initially introduced by Dembo et al. [9] and are known as inexact Newton methods. The inexact Newton method is defined by

$$
x_{n+1}=x_{n}+v_{n}, F_{x_{n}}^{\prime} v_{n}=-F\left(x_{n}\right)+y_{n}, n \in \mathbb{N}_{0},
$$

where $F$ is a continuously Fréchet differentiable nonlinear operator defined on nonempty open convex subset $D$ of Banach space $X$ with values into another Banach space $Y$ and $\left\{y_{n}\right\}$ is a sequence of elements of $Y$ which depends on $\left\{x_{n}\right\}$. The convergence behavior of method (3) depends on residual controls of $\left\{y_{n}\right\}$. Avoiding both disadvantages of Newton's method, Morini [4] has proposed the following inexact Newton-like method:

$$
x_{n+1}=x_{n}+s_{n}, B_{n} s_{n}=-F\left(x_{n}\right)+r_{n}, n \in \mathbb{N}_{0},
$$

where $\left\{r_{n}\right\}$ is a sequence in finite Banach space $Y$ while $\left\{B_{n}\right\}$ is a sequence of invertible operator from finite Banach space $X$ to $Y$ and established a local convergence result for the inexact Newton-like method (4) under the following residual controls:

$$
\left\|P_{n} r_{n}\right\| \leq \eta_{n}\left\|P_{n} F\left(x_{n}\right)\right\|, n \in \mathbb{N}_{0},
$$

where $\left\{P_{n}\right\}$ is the sequence of invertible operators from $Y$ to $X$. In [5], Li and Shen generalized the condition (5) by introducing the following condition

$$
\left\|P_{n} r_{n}\right\| \leq \eta_{n}\left\|P_{n} F\left(x_{n}\right)\right\|^{k+1}, n \in \mathbb{N}_{0},
$$

where $0 \leq k \leq 1$ and established local convergence result of order $k+1$ for an inexact Newton-like method (4). Assuming the Lipschitz continuity condition on $F_{x_{0}}^{\prime-1} F^{\prime}$ and adopting the following residual controls

$$
\left\|F_{x_{0}}^{\prime-1} y_{n}\right\| \leq \eta_{n}\left\|F_{x_{0}}^{\prime-1} F\left(x_{n}\right)\right\|^{k+1}, n \in \mathbb{N}_{0} .
$$

Shen and Li [6] established a Kantorovich-type convergence criterion for the inexact Newton method (3). Assuming the residual controls (7) with $k=1$, the $\gamma$-condition and the Smale's $\alpha$-theory have been established by Shen and Li in [7] for the inexact Newton method (3).

Recently, Xu et al. [8], Argyros and Santosh George [10] have studied semilocal convergence analysis of the inexact Newton method (3) under the residual controls (7) with $k=1$ and $F_{x_{0}}^{\prime-1} F^{\prime}$ satisfy the following weak Lipschitz condition (see $[8,10]$ ).

$$
\left\|F_{x_{0}}^{\prime-1}\left(F_{x}^{\prime}-F_{y}^{\prime}\right)\right\| \leq \int_{p(x)}^{p^{\prime}(x y)} L(u) d u
$$

for all $x \in B_{r}\left(x_{0}\right)$ and $y \in B_{(r-\rho(x))}[x]$, where $r$ is a positive real number, $p(x)=$ $=\left\|x-x_{0}\right\|, p^{\prime}(x y)=\left\|x-x_{0}\right\|+\|x-y\| \leq r$ and $L:[0, r] \rightarrow[0, \infty)$ is a nondecreasing 
integrable function.

The above discussion is based on the assumption that the involved operator is Fréchet differentiable. In various cases, the nonlinear operator may not be Fréchet differentiable, though it can be dissolved as a sum of two nonlinear operators such that one is Fréchet differentiable and second is continuous. So, we consider the following generalized operator equation:

$$
F(x)+G(x)=0,
$$

where $F, G: D \rightarrow Y$ are nonlinear operators defined on a nonempty open convex subset $D$ of a Banach space $X$ with values in another Banach space $Y$ such that $F$ is Fréchet differentiable operator at each point of $D$ and $G$ is a continuous on $D$. This brings us to the following natural question:

Question 1 Is it possible to develop an inexact Newton-like method for solving nonFréchet differentiable operator equation (9)?

The purpose of this paper is to introduce a new inexact Newton-like algorithm and discuss its semilocal convergence analysis for finding the approximate solution of generalized operator equation (9). Since our assumptions on $F$ and $G$ in generalized operator equation (9) are fairly general, our main result covers a wide variety of nonlinear operator equations. In fact, our main result provides an affirmative answer of Question 1 and also significantly improves the corresponding results of $[7,8,10]$.

\section{Preliminaries}

In this section, we give some definitions and lemmas which are useful in the main result of the paper.

Lemma 1 [10] Let $R, r, \lambda, \omega, b$ and $\rho$ be real numbers such that $0<r<R, \lambda>$ $0, \omega \geq 1, \rho \geq 0$ and $0<b \leq 1$. Let $L$ and $L_{0}$ be two positive nondecreasing integrable functions defined on any involve intervals. Define two functions $f, g:[0, R] \rightarrow \mathbb{R}$ by

$$
\begin{gathered}
f(t)=\lambda-b t+\rho t^{2}+\omega \int_{0}^{t} L(u)(t-u) d u, 0 \leq t \leq R \\
g(t)=\lambda-t+\omega \int_{0}^{t} L_{0}(u)(t-u) d u, 0 \leq t \leq R .
\end{gathered}
$$

Let $r_{b}, s_{b}$ and $\sigma$ be real numbers which are given by:

$$
\begin{gathered}
r_{b}=\sup \left\{r \in(0, R): \omega \int_{0}^{r} L(u) d u+2 \rho r \leq b\right\}, \\
s_{b}=b r_{b}-\rho r_{b}^{2}-\omega \int_{0}^{r_{b}} L(u)\left(r_{b}-u\right) d u
\end{gathered}
$$


and $\sigma=\omega \int_{0}^{R} L(u) d u+2 \rho R$. Then, we have the following:

(a) $r_{b}=\left\{\begin{array}{l}R, \quad \text { if } \sigma<b, \\ r_{b}^{\prime}, \quad \text { if } \sigma \geq b,\end{array}\right.$ where $r_{b}^{\prime} \in[0, R]$ is such that $\omega \int_{0}^{r_{b}^{\prime}} L(u) d u+2 \rho r_{b}^{\prime}=b$.

(b) $s_{b} \geq \omega \int_{0}^{r_{b}^{\prime}} L(u) u d u+\rho\left(r_{b}^{\prime}\right)^{2}$ if $\sigma<b$ and $s_{b}=\omega \int_{0}^{r_{b}^{\prime}} L(u) u d u+\rho\left(r_{b}^{\prime}\right)^{2}$ if $\sigma \geq b$.

Lemma 2 Suppose all the assumptions of Lemma 1 hold, $\lambda \leq s_{b}$ and $L_{0}(t) \leq L(t)$ for each $t \in[0, r]$. Then, we have the following:

(a) The function $f$ is strictly decreasing on $\left[0, r_{b}\right]$ and has a unique zero $t^{*} \in\left[0, r_{b}\right]$ satisfying $\lambda<t^{*}$.

(b) The sequence $\left\{t_{n}\right\}$ generated by

$$
t_{0}=0, t_{n+1}=t_{n}-\frac{f\left(t_{n}\right)}{g^{\prime}\left(t_{n}\right)}, n \in \mathbb{N}
$$

is strictly increasing and converges to $t^{*}$. Moreover,

$$
t_{n+1}<t_{n}<t^{*}, n \in \mathbb{N}_{0} \text {. }
$$

Proof: We shall prove this lemma by method of mathematical induction. One can easily observe that $0=t_{0}<t_{1}=\lambda<t^{*}$. Let $k>1$ be positive integer. Assume that the following hold for $n=1,2,3, \cdots, k$

$$
t_{k}>t_{k-1} .
$$

Since $g^{\prime \prime}(t)=\omega L_{0}(t)>0$, this implies that $-g^{\prime}$ is strictly decreasing on $\left[0, r_{b}\right]$. Hence

$$
-g^{\prime}\left(t_{k}\right)>-g^{\prime}\left(t^{*}\right) \geq-g^{\prime}\left(r_{b}\right)=1-\omega \int_{0}^{r_{b}} L_{0}(u) d u>1-\omega \int_{0}^{r_{b}} L(u) d u>0 .
$$

Note that $f\left(t_{k}\right)>0$ on $\left[0, t^{*}\right]$. It follows that

$$
t_{k+1}=t_{k}-\frac{f\left(t_{k}\right)}{g^{\prime}\left(t_{k}\right)}>t_{k}
$$

Thus (15) hold for $n=k+1$. Therefore, by mathematical induction (15) is true for all $n \in \mathbb{N}_{0}$.

Define a function $\mathscr{N}$ on $\left[0, t^{*}\right]$ by

$$
\mathscr{N}(t)=t-\frac{f(t)}{g^{\prime}(t)} .
$$

Since $g^{\prime}(t)<0$ on $\left[0, t^{*}\right]$ and $f\left(t^{*}\right)=0$, which implies $\lim _{t \rightarrow t^{*}} \frac{f(t)}{g^{\prime}(t)}=0$ and $\mathscr{N}\left(t^{*}\right)=t^{*}$. Thus the function $\mathscr{N}(t)$ is well defined and continuous on $\left[0, t^{*}\right]$. Now for each 
$t \in\left[0, t^{*}\right]$, we have

$$
\mathscr{N}^{\prime}(t)=\frac{-g^{\prime}(t)\left(f^{\prime}(t)-g^{\prime}(t)\right)+f(t) g^{\prime \prime}(t)}{\left(g^{\prime}(t)\right)^{2}}>0 .
$$

Thus $\mathscr{N}(t)$ is monotonically increasing on $\left[0, t^{*}\right]$ which implies that

$$
t_{n}<t_{n+1}=\mathscr{N}\left(t_{n}\right)<\mathscr{N}\left(t^{*}\right)=t^{*} .
$$

This completes the proof.

Lemma 3 Let $X$ and $Y$ be two Banach spaces, $D$ a nonempty open subset of $X$ and $A(x) \in B(X, Y), x \in D$ such that $A\left(x_{0}\right)^{-1}$ exists for some $x_{0} \in D$. Let $r>0$ be such that $B_{r}\left(x_{0}\right) \subset D$ and $L_{0}:[0, r] \rightarrow \mathbb{R}$ a positive nondecreasing integrable function. Assume that the following conditions hold:

(C1) $\left\|A\left(x_{0}\right)^{-1}\left(A(x)-A\left(x_{0}\right)\right)\right\| \leq \int_{0}^{\left\|x-x_{0}\right\|} L_{0}(u) d u$ for all $x \in B_{r}\left(x_{0}\right)$;

(C2) $\int_{0}^{r} L_{0}(u) d u<1$.

Then, for all $x \in B_{r}\left(x_{0}\right), A(x)$ is invertible and

$$
\left\|A(x)^{-1} A\left(x_{0}\right)\right\| \leq \frac{1}{1-\int_{0}^{\left\|x-x_{0}\right\|} L_{0}(u) d u} \leq-\frac{1}{g^{\prime}\left(\left\|x-x_{0}\right\|\right)} .
$$

Proof: Let $x \in B_{r}\left(x_{0}\right)$. Then

$$
\begin{aligned}
\left\|I-A\left(x_{0}\right)^{-1} A(x)\right\| & =\left\|A\left(x_{0}\right)^{-1}\left(A(x)-A\left(x_{0}\right)\right)\right\| \\
& \leq \int_{0}^{\left\|x-x_{0}\right\|} L_{0}(u) d u<1 .
\end{aligned}
$$

By Banach Lemma, we have

$$
\left\|A(x)^{-1} A\left(x_{0}\right)\right\| \leq \frac{1}{1-\int_{0}^{\left\|x-x_{0}\right\|} L_{0}(u) d u} .
$$

Note

$$
-g^{\prime}\left(\left\|x-x_{0}\right\|\right)=1-\omega \int_{0}^{\left\|x-x_{0}\right\|} L_{0}(u) d u \leq 1-\int_{0}^{\left\|x-x_{0}\right\|} L_{0}(u) d u .
$$

This implies that

$$
\frac{1}{1-\int_{0}^{\left\|x-x_{0}\right\|} L_{0}(u) d u} \leq-\frac{1}{g^{\prime}\left(\left\|x-x_{0}\right\|\right)} .
$$

Thus, (17) follows from (18) and (19). This completes the proof. 


\section{Semilocal convergence analysis}

In this section, we propose a new inexact Newton-like method and study its semilocal convergence analysis.

Algorithm 1 Let $X$ and $Y$ be two Banach spaces, $D$ a nonempty open convex subset of $X$ and $x_{0} \in D$. Let $F: D \rightarrow Y$ be a nonlinear Fréchet differentiable operator and $G: D \rightarrow Y$ is a continuous operator and $A(x) \in B(X, Y)$ an approximation of $F_{x}^{\prime}$ for $x \in D$ such that $A\left(x_{0}\right)^{-1}$ exists. Let $x_{0}$ be an initial guess. For the given residual $r_{n}$ and the iteration $x_{n}$, we defined the next iterate $x_{n+1}$ as follows:

$$
x_{n+1}=x_{n}+s_{n}, A\left(x_{n}\right) s_{n}=-\left(F\left(x_{n}\right)+G\left(x_{n}\right)\right)+r_{n}, n \in \mathbb{N}_{0},
$$

where $\left\{r_{n}\right\} \subset Y$ and in general depends on $\left\{x_{n}\right\}$.

Let $\left\{r_{n}\right\}$ satisfy the following residual controls:

$$
\left\|A\left(x_{0}\right)^{-1} r_{n}\right\| \leq \eta_{n}\left\|A\left(x_{0}\right)^{-1}\left(F\left(x_{n}\right)+G\left(x_{n}\right)\right)\right\|^{2}, \quad n \in \mathbb{N}_{0},
$$

where $\left\{\eta_{n}\right\}$ is the sequence of forcing terms such that $0 \leq \eta_{n}<1$ for all $n \in \mathbb{N}_{0}$ with $\eta=\sup \left\{\eta_{n}\right\}$.

Now we shall discuss the semilocal convergence analysis of Algorithm 1 under the condition (21) and the following weak Lipschitz condition:

$$
\left\|A\left(x_{0}\right)^{-1}\left(F_{x}^{\prime}-F_{y}^{\prime}\right)\right\| \leq \int_{p(x)}^{p^{\prime}(x y)} L(u) d u
$$

for all $x \in B_{r}\left(x_{0}\right)$ and $y \in B_{r-p(x)}\left[x_{0}\right]$, where $p(x)=\left\|x-x_{0}\right\|, p^{\prime}(x y)=\left\|x-x_{0}\right\|+$ $\|x-y\| \leq r$ and $L:[0, r] \rightarrow \mathbb{R}$ is a positive nondecreasing integrable function.

Theorem 1 Let $X$ and $Y$ be two Banach spaces, $D$ a nonempty open convex subset of $X$ and $x_{0} \in D$. Let $F: D \rightarrow Y$ be a nonlinear Fréchet differentiable operator and $G: D \rightarrow Y$ is a continuous operator and $A(x) \in B(X, Y)$ an approximation of $F_{x}^{\prime}$ for $x \in D$ such that $A\left(x_{0}\right)^{-1}$ exists. Suppose that all the assumptions of Lemma 2 hold. Assume that (C1), (C2), (21), (22) and the following conditions hold:

(C3) $\left\|A\left(x_{0}\right)^{-1}\left(F\left(x_{0}\right)+G\left(x_{0}\right)\right)\right\|=\beta$ for some $\beta>0$;

(C4) $\left\|A\left(x_{0}\right)^{-1}(G(x)-G(y))\right\| \leq K\|x-y\|$ for all $x, y \in D$ and for some $K \geq 0$;

(C5) $\left\|A\left(x_{0}\right)^{-1}\left(A(x)-F_{x}^{\prime}\right)\right\|<\delta$ for all $x \in D$ and for some $\delta \geq 0$;

(C6) $\sqrt{\eta}\left\|A\left(x_{0}\right)^{-1}\left(F\left(x_{0}\right)+G\left(x_{0}\right)\right)\right\| \leq 1$;

(C7) $\rho=\eta \omega \frac{\left(1+\int_{0}^{R} L_{0}(u) d u\right)^{2}}{(1-\sqrt{\eta})^{2}}, \kappa=K+\delta, \omega=1+\sqrt{\eta}, b=1-(1+\sqrt{\eta}) \kappa$. 
Assume that $\lambda=\omega \beta \leq \min \left\{1 / \sqrt{\eta}, s_{b}\right\}$ and $B_{t^{*}}\left[x_{0}\right] \subseteq B_{R}\left(x_{0}\right)$. Then, the sequence $\left\{x_{n}\right\}$ generated by inexact Newton-like method (1) is well defined, remains in $B_{t^{*}}\left[x_{0}\right]$ and the following assertions hold:

(a) $\left\|A\left(x_{n}\right)^{-1} A\left(x_{0}\right)\right\| \leq-g^{\prime}\left(t_{n}\right)^{-1}, n \in \mathbb{N}$;

(b) $\sqrt{\eta}\left\|A\left(x_{0}\right)^{-1}\left(F\left(x_{n}\right)+G\left(x_{n}\right)\right)\right\| \leq 1, n \in \mathbb{N}$;

(c) $\left\|x_{n+1}-x_{n}\right\| \leq t_{n+1}-t_{n}, n \in \mathbb{N}_{0}$, where $\left\{t_{n}\right\}$ is a real sequence given by (14).

(d) The sequence $\left\{x_{n}\right\}$ converges to the solution $x^{*} \in B_{t^{*}}\left[x_{0}\right]$ of equation (9) with the following estimate:

$$
\left\|x^{*}-x_{n}\right\| \leq t^{*}-t_{n}, n \in \mathbb{N}_{0} .
$$

Proof: Note that $\lambda \leq \min \left\{1 / \sqrt{\eta}, s_{b}\right\}$. Thus, Lemma 2 is applicable. Hence the sequence $\left\{t_{n}\right\}$ generated by (14) is strictly increasing and converges to $t^{*}$. For $x_{0} \in D$, using Algorithm 1, (C3), (C6), (21) and (14), we have

$$
\begin{aligned}
\left\|x_{1}-x_{0}\right\| & \leq\left\|A\left(x_{0}\right)^{-1}\left(F\left(x_{0}\right)+G\left(x_{0}\right)\right)\right\|+\left\|A\left(x_{0}\right)^{-1} r_{0}\right\| \\
& =(1+\sqrt{\eta})\left\|A\left(x_{0}\right)^{-1}\left(F\left(x_{0}\right)+G\left(x_{0}\right)\right)\right\| \leq(1+\sqrt{\eta}) \beta=t_{1} \leq t^{*}
\end{aligned}
$$

Thus, $x_{1} \in B_{t^{*}}\left(x_{0}\right)$. We now proceed with the following steps:

Step I: $(a)-(c)$ hold for $n=1$.

From (16), we have

$$
\left\|A\left(x_{1}\right)^{-1} A\left(x_{0}\right)\right\| \leq-g^{\prime}\left(t_{1}\right)^{-1} .
$$

Using Algorithm 1 and (21), we have

$$
\begin{aligned}
& \left\|A\left(x_{0}\right)^{-1}\left(F\left(x_{1}\right)+G\left(x_{1}\right)\right)\right\| \\
= & \left\|A\left(x_{0}\right)^{-1}\left(F\left(x_{1}\right)+G\left(x_{1}\right)-F\left(x_{0}\right)-G\left(x_{0}\right)-A\left(x_{0}\right)\left(x_{1}-x_{0}\right)\right)+A\left(x_{0}\right)^{-1} r_{0}\right\| \\
& +A\left(x_{0}\right)^{-1} r_{0}+A\left(x_{0}\right)^{-1}\left(G\left(x_{1}\right)-G\left(x_{0}\right)\right) \| \\
\leq & \int_{0}^{1} \int_{0}^{t\left\|x_{1}-x_{0}\right\|} L(u) d u\left\|x_{1}-x_{0}\right\| d t+\eta\left\|A\left(x_{0}\right)^{-1}\left(F\left(x_{0}\right)+G\left(x_{0}\right)\right)\right\|^{2}+\kappa\left\|x_{1}-x_{0}\right\|
\end{aligned}
$$

Again, by using Algorithm 1, we have

$$
\begin{aligned}
& \left\|A\left(x_{0}\right)^{-1} A\left(x_{0}\right)\left(x_{1}-x_{0}\right)\right\| \geq\left\|A\left(x_{0}\right)^{-1}\left(F\left(x_{0}\right)+G\left(x_{0}\right)\right)\right\|-\left\|A\left(x_{0}\right)^{-1} r_{0}\right\| \text { and } \\
& \qquad \begin{aligned}
\left\|x_{1}-x_{0}\right\| & \geq\left\|A\left(x_{0}\right)^{-1}\left(F\left(x_{0}\right)+G\left(x_{0}\right)\right)\right\|-\sqrt{\eta}\left\|A\left(x_{0}\right)^{-1}\left(F\left(x_{0}\right)+G\left(x_{0}\right)\right)\right\| \\
& \geq(1-\sqrt{\eta})\left\|A\left(x_{0}\right)^{-1}\left(F\left(x_{0}\right)+G\left(x_{0}\right)\right)\right\| .
\end{aligned}
\end{aligned}
$$


Using (24), we have

$$
\begin{aligned}
& (1+\sqrt{\eta})\left\|A\left(x_{0}\right)^{-1}\left(F\left(x_{1}\right)+G\left(x_{1}\right)\right)\right\| \\
= & (1+\sqrt{\eta})\left(\int_{0}^{\left\|x_{1}-x_{0}\right\|} L(u)\left(\left\|x_{1}-x_{0}\right\|-u\right) d u+\frac{\eta}{(1-\sqrt{\eta})^{2}}\left\|x_{1}-x_{0}\right\|^{2}+\kappa\left\|x_{1}-x_{0}\right\|\right) \\
\leq & \omega \int_{0}^{t_{1}} L(u)\left(t_{1}-u\right) d u+\rho t_{1}^{2}+(1+\sqrt{\eta}) \kappa\left(t_{1}-t_{0}\right)=f\left(t_{1}\right) .
\end{aligned}
$$

Note that $f$ is decreasing on $\left[0, t^{*}\right]$. This implies that

$$
(1+\sqrt{\eta})\left\|A\left(x_{0}\right)^{-1}\left(F\left(x_{1}\right)+G\left(x_{1}\right)\right)\right\| \leq f\left(t_{1}\right) \leq f\left(t_{0}\right)=\lambda \text { and }
$$

$$
\sqrt{\eta}\left\|A\left(x_{0}\right)^{-1}\left(F\left(x_{1}\right)+G\left(x_{1}\right)\right)\right\| \leq \frac{\lambda \sqrt{\eta}}{1+\sqrt{\eta}}=\sqrt{\eta}\left\|A\left(x_{0}\right)^{-1}\left(F\left(x_{0}\right)+G\left(x_{0}\right)\right)\right\| \leq 1 .
$$

From Algorithm 1, (23) and (25), we have

$$
\begin{aligned}
\left\|x_{2}-x_{1}\right\| & \leq\left\|A\left(x_{1}\right)^{-1} A\left(x_{0}\right)\right\|\left(\left\|A\left(x_{0}\right)^{-1}\left(F\left(x_{1}\right)+G\left(x_{1}\right)\right)\right\|+\left\|A\left(x_{0}\right)^{-1} r_{1}\right\|\right) \\
& =(1+\sqrt{\eta})\left\|A\left(x_{1}\right)^{-1} A\left(x_{0}\right)\right\|\left\|A\left(x_{0}\right)^{-1}\left(F\left(x_{1}\right)+G\left(x_{1}\right)\right)\right\| \\
& =-\frac{f\left(t_{1}\right)}{g^{\prime}\left(t_{1}\right)}=t_{2}-t_{1}
\end{aligned}
$$

and $\left\|x_{2}-x_{0}\right\| \leq\left\|x_{2}-x_{1}\right\|+\left\|x_{1}-x_{0}\right\| \leq t_{2} \leq t^{*}$. Thus, $x_{2} \in B_{t^{*}}\left(x_{0}\right)$.

Step II: $(a)-(c)$ hold and $x_{n} \in B_{t^{*}}\left(x_{0}\right)$ for all $n \in \mathbb{N}$.

Let $k \geq 1$ be a positive integer. Assume that $(a)-(c)$ hold for $n=1,2, \cdots, k$.

Using triangular inequality, we have

$$
\left\|x_{k+1}-x_{0}\right\| \leq\left\|x_{k+1}-x_{k}\right\|+\left\|x_{k}-x_{0}\right\| \leq t_{k+1}-t_{0} \leq t^{*} .
$$

This implies that $x_{k+1} \in B_{t^{*}}\left[x_{0}\right]$. From Lemma 3, we have

$$
\left\|A\left(x_{k+1}\right)^{-1} A\left(x_{0}\right)\right\| \leq-g^{\prime}\left(t_{k+1}\right)^{-1} .
$$

Using Algorithm 1, we get

$$
\begin{aligned}
& \left\|A\left(x_{0}\right)^{-1}\left(F\left(x_{k+1}\right)+G\left(x_{k+1}\right)\right)\right\| \\
= & \left\|A\left(x_{0}\right)^{-1}\left(F\left(x_{k+1}\right)+G\left(x_{k+1}\right)-F\left(x_{k}\right)-G\left(x_{k}\right)-A\left(x_{k}\right)\left(x_{k+1}-x_{k}\right)\right)+A\left(x_{0}\right)^{-1} r_{k}\right\| \\
\leq & \int_{0}^{1} \int_{\left\|x_{k}-x_{0}\right\|}^{\left\|x_{k}-x_{0}\right\|+t\left\|x_{k+1}-x_{k}\right\|} L(u) d u\left\|x_{k+1}-x_{k}\right\| d t+\kappa\left\|x_{k+1}-x_{k}\right\|+\left\|A\left(x_{0}\right)^{-1} r_{k}\right\| \\
= & \int_{0}^{\left\|x_{k+1}-x_{k}\right\|}\left(\left\|x_{k+1}-x_{k}\right\|-v\right) L\left(\left\|x_{k}-x_{0}\right\|+v\right) d v+\kappa\left\|x_{k+1}-x_{k}\right\|+\left\|A\left(x_{0}\right)^{-1} r_{k}\right\| .
\end{aligned}
$$

Again using Algorithm 1, we have

$$
\begin{aligned}
\left\|A\left(x_{0}\right)^{-1} A\left(x_{k}\right)\left(x_{k+1}-x_{k}\right)\right\| & =\left\|A\left(x_{0}\right)^{-1}\left(F\left(x_{k}\right)+G\left(x_{k}\right)\right)\right\|-\left\|A\left(x_{0}\right)^{-1} r_{k}\right\| \\
& \geq(1-\sqrt{\eta})\left\|A\left(x_{0}\right)^{-1}\left(F\left(x_{k}\right)+G\left(x_{k}\right)\right)\right\|,
\end{aligned}
$$


which implies that

$$
\left\|A\left(x_{0}\right)^{-1}\left(F\left(x_{k}\right)+G\left(x_{k}\right)\right)\right\| \leq \frac{\left\|A\left(x_{0}\right)^{-1} A\left(x_{k}\right)\right\|\left\|x_{k+1}-x_{k}\right\|}{1-\sqrt{\eta}} .
$$

Now

$$
\begin{aligned}
\left\|A\left(x_{0}\right)^{-1} A\left(x_{k}\right)\right\| & =\left\|I+A\left(x_{0}\right)^{-1}\left(A\left(x_{k}\right)-A\left(x_{0}\right)\right)\right\| \\
& \leq 1+\int_{0}^{\left\|x_{k}-x_{0}\right\|} L_{0}(u) d u \leq 1+\int_{0}^{t_{k}} L_{0}(u) d u .
\end{aligned}
$$

Using (27) and (28), we have

$$
\begin{aligned}
& (1+\sqrt{\eta})\left\|A\left(x_{0}\right)^{-1}\left(F\left(x_{k+1}\right)+G\left(x_{k+1}\right)\right)\right\| \\
\leq & \omega \int_{0}^{\left\|x_{k+1}-x_{k}\right\|}\left(\left\|x_{k+1}-x_{k}\right\|-v\right) L\left(\left\|x_{k}-x_{0}\right\|+v\right) d v+(1+\sqrt{\eta}) \kappa\left\|x_{k+1}-x_{k}\right\| \\
+ & \eta(1+\sqrt{\eta}) \frac{\left(1+\int_{0}^{R} L_{0}(u) d u\right)^{2}}{(1-\sqrt{\eta})^{2}}\left\|x_{k+1}-x_{k}\right\|^{2} \\
\leq & \omega \int_{0}^{t_{k+1}-t_{k}}\left(t_{k+1}-t_{k}-v\right) L\left(t_{k}+v\right) d v+(1+\sqrt{\eta}) \kappa\left(t_{k+1}-t_{k}\right)+\rho\left(t_{k+1}-t_{k}\right)^{2} \\
= & \omega \int_{0}^{t_{k+1}}\left(t_{k+1}-u\right) L(u) d u-\omega \int_{0}^{t_{k}}\left(t_{k}-u\right) L(u) d u-\omega \int_{0}^{t_{k}} L(u)\left(t_{k+1}-t_{k}\right) d u \\
+ & \rho\left(t_{k+1}^{2}-t_{k}^{2}-2 t_{k}\left(t_{k+1}-t_{k}\right)\right)+(1+\sqrt{\eta}) \kappa\left(t_{k+1}-t_{k}\right) \\
\leq & f\left(t_{k+1}\right) .
\end{aligned}
$$

Since $f$ is decreasing on $\left[0, t^{*}\right]$, then

$$
\begin{aligned}
& (1+\sqrt{\eta})\left\|A\left(x_{0}\right)^{-1}\left[F\left(x_{k+1}\right)+G\left(x_{k+1}\right)\right]\right\| \leq f\left(t_{k+1}\right) \leq f\left(t_{0}\right)=\lambda \text { and } \\
& \sqrt{\eta}\left\|A\left(x_{0}\right)^{-1}\left(F\left(x_{k+1}\right)+G\left(x_{k+1}\right)\right)\right\| \leq \frac{\lambda \sqrt{\eta}}{1+\sqrt{\eta}}=\sqrt{\eta}\left\|A\left(x_{0}\right)^{-1}\left(F\left(x_{0}\right)+G\left(x_{0}\right)\right)\right\|<1 .
\end{aligned}
$$

Using Lemma 3 and Algorithm 1, we have

$$
\begin{aligned}
& \left\|x_{k+2}-x_{k+1}\right\| \\
\leq & \left\|A\left(x_{k+1}\right)^{-1} A\left(x_{0}\right)\right\|\left(\left\|A\left(x_{0}\right)^{-1}\left(F\left(x_{k+1}\right)+G\left(x_{k+1}\right)\right)\right\|+\left\|A\left(x_{0}\right)^{-1} r_{k+1}\right\|\right) \\
\leq & (1+\sqrt{\eta})\left\|A\left(x_{k+1}\right)^{-1} A\left(x_{0}\right)\right\|\left\|A\left(x_{0}\right)^{-1}\left(F\left(x_{k+1}\right)+G\left(x_{k+1}\right)\right)\right\| \\
\leq & -\frac{f\left(t_{k+1}\right)}{g^{\prime}\left(t_{k+1}\right)}=t_{k+2}-t_{k+1}
\end{aligned}
$$

this implies $\left\|x_{k+2}-x_{0}\right\| \leq\left\|x_{k+2}-x_{k+1}\right\|+\left\|x_{k+1}-x_{0}\right\| \leq t_{k+2} \leq t^{*}$.

Therefore, $(a)-(c)$ hold for $n=k+1$. Hence, by mathematical induction $(a)-(c)$ is true for all $n \in \mathbb{N}$. 
(d) For $m, n \in \mathbb{N}_{0}$, using triangular inequality, we have

$$
\left\|x_{n+m}-x_{n}\right\| \leq \sum_{k=n}^{m+n-1}\left\|x_{k+1}-x_{k}\right\| \leq \sum_{k=n}^{m+n-1}\left(t_{k+1}-t_{k}\right)=t_{m+n}-t_{n} .
$$

Taking $n=0$ in (31), we get $\left\|x_{m}-x_{0}\right\| \leq t_{m}-t_{0} \leq t^{*}, m \in \mathbb{N}_{0}$.

Thus, $x_{m} \in B_{t^{*}}\left(x_{0}\right)$. By the definition of majorizing sequence, sequence $\left\{x_{n}\right\}$ must be a Cauchy sequence, and it converges to $x^{*}$. Taking limit as $m \rightarrow \infty$ in (31), we have

$$
\left\|x^{*}-x_{n}\right\| \leq t^{*}-t_{n}
$$

This completes the proof.

If we take $A(x)=F_{x}^{\prime}, x \in D$, then inexact Newton-like method (1) reduces to

$$
x_{n+1}=x_{n}+s_{n}, \quad F_{x_{n}}^{\prime} s_{n}=-\left(F\left(x_{n}\right)+G\left(x_{n}\right)\right)+r_{n}, n \in \mathbb{N}_{0},
$$

where $\left\{r_{n}\right\}$ is a sequence in $Y$ which is generally dependent on $\left\{x_{n}\right\}$. Assume that sequence $\left\{r_{n}\right\}$ satisfies the following residual controls:

$$
\left\|F_{x_{0}}^{\prime-1} r_{n}\right\| \leq \eta_{n}\left\|F_{x_{0}}^{\prime-1}\left(F\left(x_{n}\right)+G\left(x_{n}\right)\right)\right\|^{2}, n \in \mathbb{N}_{0},
$$

where $\left\{\eta_{n}\right\}$ is the sequence of forcing terms such that $\eta=\sup \left\{\eta_{n}\right\}<1$ and $F_{x_{0}}^{\prime-1} F^{\prime}$ satisfies the weak Lipschitz condition (22) with $A\left(x_{0}\right)=F_{x_{0}}^{\prime}$.

Corollary 1 Let $X$ and $Y$ be two Banach spaces, $D$ a nonempty open convex subset of $X, x_{0} \in D$. Let $F: D \rightarrow Y$ be Fréchet differentiable operator such that $F_{x_{0}}^{\prime-1}$ exists and $G: D \rightarrow Y$ a continuous operator. Suppose that all the assumptions of Lemma 2 hold and $F$ satisfies (22), (C1), (C3), (C4), (C6) with $A\left(x_{0}\right)=F_{x_{0}}^{\prime},(33),(C 2),(C 7)$ with $\delta=0$ and assume that $\lambda=\omega \beta \leq \min \left\{\frac{1}{\sqrt{\eta}}, s_{b}\right\}$. Then, the sequence $\left\{x_{n}\right\}$ generated by (32) is well defined, remains in $B_{t^{*}}\left[x_{0}\right]$ and for each $n \in \mathbb{N}_{0}$ the following assertions hold:

(a) $\left\|F_{x_{n}}^{\prime-1} F_{x_{0}}^{\prime}\right\| \leq-g^{\prime}\left(t_{n}\right)^{-1}$, (b) $\sqrt{\eta}\left\|F_{x_{0}}^{\prime-1}\left(F\left(x_{n}\right)+G\left(x_{n}\right)\right)\right\| \leq 1$,

(c) $\left\|x_{n+1}-x_{n}\right\| \leq t_{n+1}-t_{n}$, where $\left\{t_{n}\right\}$ is a real sequence generated by (14) and

(d) The sequence $\left\{x_{n}\right\}$ converges to the solution $x^{*} \in B_{t^{*}}\left[x_{0}\right]$ of equation (9). Moreover,

$$
\left\|x^{*}-x_{n}\right\| \leq t^{*}-t_{n}, n \in \mathbb{N}_{0} .
$$

\section{Kantorovich type condition and $\gamma$-condition}

In this section, we show that some well known results are the special cases of our main result.

Set $A(x)=F_{x}^{\prime}$ for all $x \in D, \eta_{n}=0$ for all $n \in \mathbb{N}_{0}$ and $L(t)=L$ for all $t \geq 0$ a positive constant in Theorem 1, we have the following result: 
Corollary 2 Let $X$ and $Y$ be two Banach spaces, $D$ a nonempty open convex subset of $X, x_{0} \in D$. Let $F: D \rightarrow Y$ be Fréchet differentiable operator such that $F_{x_{0}}^{\prime-1}$ exists and $G: D \rightarrow Y$ a continuous operator. Assume that $F$ satisfies $(C 3),(C 4)$ with $A\left(x_{0}\right)=F_{x_{0}}^{\prime}$ and the following conditions:

(C8) $\left\|F_{x_{0}}^{\prime-1}\left(F_{x}^{\prime}-F_{y}^{\prime}\right)\right\| \leq L\|x-y\|$ for all $x, y \in D$ and for some $L \geq 0$.

(C9) $h=\frac{\beta L}{(1-K)^{2}} \leq \frac{1}{2}$.

Let $B_{v^{*}}\left[x_{0}\right] \subset D$, where $v^{*}=\frac{1-\sqrt{1-2 h}}{h} \frac{\beta}{1-K}$. Then, the sequence $\left\{x_{n}\right\}$ generated by Newton-like method

$$
x_{n+1}=x_{n}-F_{x_{n}}^{\prime-1}\left(F\left(x_{n}\right)+G\left(x_{n}\right), n \in \mathbb{N}_{0}\right.
$$

is well defined, remains in $B_{v^{*}}\left[x_{0}\right]$ and converges to the solution of equation (9).

Proof: Set $L(t)=L$ for all $t \geq 0, \eta_{n}=0$ for all $n \in \mathbb{N}_{0}$ and $A(x)=F_{x}^{\prime}$ for all $x \in D$ in Theorem 1 , we obtain $\delta=0, \eta=0, b=1-K$ and $\omega=1$. Thus, equations (10) and (11) reduces to

$$
f(t)=\frac{L t^{2}}{2}-(1-K) t+\beta \text { and } g(t)=\frac{L t^{2}}{2}-t+\beta
$$

respectively. One can easily observe from (12) and (13) that $r_{b}=\frac{1-K}{L}$ and $s_{b}=\frac{(1-K)^{2}}{2 L}$. The assumption $(C 9)$ gives the convergence criterion $\beta=\lambda \leq s_{b}$. Thus the equation $f(t)=0$ has a solution $v^{*}=\frac{1-\sqrt{1-2 h}}{h} \frac{\beta}{1-K}$ such that $v^{*} \leq s_{b}=\frac{(1-K)^{2}}{2 L}$.

Thus, all the assumptions of Theorem 1 are satisfied. Therefore, the proof of this Corollary follows from Theorem 1 . This completes the proof.

Let $0<\gamma_{0} \leq \gamma$ and $F: D \rightarrow Y$ have continuous second Fréchet derivative and satisfy the $\gamma$-condition $[3,6,7]$

$$
\left\|F_{x_{0}}^{\prime-1} F^{\prime \prime}(x)\right\| \leq \frac{2 \gamma}{\left(1-\gamma\left\|x-x_{0}\right\|\right)^{3}}
$$

for all $x \in B_{r}\left(x_{0}\right), 0<r<\frac{1}{\gamma}$. Define

$$
L(t)=\frac{2 \gamma}{(1-\gamma t)^{3}}, 0<t<\frac{1}{\gamma} \text { and } L_{0}(t)=\frac{2 \gamma_{0}}{\left(1-\gamma_{0} t\right)^{3}}, 0<t<\frac{1}{\gamma_{0}} .
$$

Clearly $L_{0}(t) \leq L(t)$ for all $0<t<\frac{1}{\gamma}$ and functions $f$ and $g$ defined by (10) and (11) reduces to 
$f_{\gamma}(t)=\lambda-b t+\rho t^{2}+\frac{\omega \gamma t^{2}}{1-\gamma t}, 0<t<\frac{1}{\gamma}$ and $g_{\gamma_{0}}(t)=\lambda-t+\frac{\omega \gamma_{0} t^{2}}{1-\gamma_{0} t}, 0<t<\frac{1}{\gamma_{0}}$ respectively. One cane easily observe from (12) and (13) that $r_{b}$ and $s_{b}$ satisfy

$$
\omega\left(\frac{1}{\left(1-\gamma r_{b}\right)^{2}}-1\right)+\rho r_{b}=b \text { and } s_{b}=\frac{\gamma r_{b}^{2}}{\left(1-\gamma r_{b}\right)^{2}} \text {. }
$$

Then the corresponding convergence criterion of Theorem 1 becomes

$$
\left\|A\left(x_{0}\right)^{-1}\left(F\left(x_{0}\right)+G\left(x_{0}\right)\right)\right\| \leq \frac{\gamma r_{b}^{2}}{\left(1-\gamma r_{b}\right)^{2}} .
$$

In the special case when $\eta_{n}=0$ for all $n \in \mathbb{N}_{0}$, then $\eta=0$ and $C(7)$ implies that $\rho=0, \kappa=K+\delta, b=1-\kappa, \omega=1$. From (36), we have

$$
r_{b}=\left(1-\frac{1}{\sqrt{1+b}}\right) \frac{1}{\gamma} \text { and } s_{b}=\frac{1+b}{\gamma}\left(1-\frac{1}{\sqrt{1+b}}\right)^{2} .
$$

Corollary 3 Let $X$ and $Y$ be two Banach spaces, $D$ a nonempty open convex subset of $X$ and $x_{0} \in D$. Let $F: D \rightarrow Y$ be a nonlinear Fréchet differentiable operator and $G: D \rightarrow Y$ is a continuous operator and $A(x) \in B(X, Y)$ an approximation of $F_{x}^{\prime}$ for $x \in D$ such that $A\left(x_{0}\right)^{-1}$ exists. Assume that $(C 3)-(C 5)$ and the following conditions hold:

(C10) $\left\|A\left(x_{0}\right)^{-1}\left(A(x)-A\left(x_{0}\right)\right)\right\| \leq \frac{1}{\left(1-\gamma_{0}\left\|x-x_{0}\right\|\right)^{2}}-1$;

(C11) $\left\|A\left(x_{0}\right)^{-1}\left(F_{x}^{\prime}-F_{y}^{\prime}\right)\right\| \leq \frac{1}{\left(1-\gamma\left\|x-x_{0}\right\|-\gamma\|x-y\|\right)^{2}}-\frac{1}{\left(1-\gamma\left\|x-x_{0}\right\|\right)^{2}}$

for all $x, y \in B_{s^{*}}\left[x_{0}\right]$ and $\left\|x-x_{0}\right\|+\|x-y\| \leq s^{*}$, where

$$
s^{*}=\frac{b+\lambda \gamma-\sqrt{(b+\lambda \gamma)^{2}-4 \lambda \gamma(1+b)}}{2(1+b) \gamma} .
$$

Let $\beta \gamma \leq 2+b-2 \sqrt{1+b}$. Then the sequence generated by Newton-like method

$$
x_{n+1}=x_{n}-A\left(x_{n}\right)^{-1}\left[F\left(x_{n}\right)+G\left(x_{n}\right)\right], n \in \mathbb{N}_{0}
$$

is well defined, remains in $B_{s^{*}}\left[x_{0}\right]$ and converges to the solution $x^{*}$ of operator equation (9). Moreover, $\left\|x_{n+1}-x_{n}\right\| \leq s_{n+1}-s_{n}$ and $\left\|x^{*}-x_{n}\right\| \leq s^{*}-s_{n}, n \in \mathbb{N}_{0}$, where $\left\{s_{n}\right\}$ is real sequence generated by

$$
s_{0}=0, s_{n+1}=s_{n}-\frac{f_{\gamma}\left(s_{n}\right)}{g_{\gamma_{0}}^{\prime}\left(s_{n}\right)}, n \in \mathbb{N} .
$$

Proof: If we take L and $L_{0}$ defined by (35) and $\eta=0$ in Theorem 1, then the proof of Corollary 3 follows from Theorem 1. 


\section{Remarks}

(1) If $L_{0}=L$ and $G=0$, Corollary 1 reduces to [8, Theorem 3.2].

(2) Corollary 1 is an improvement over the [8, Theorem 3.2] in the sense of larger convergence domain and tighter error bounds.

(3) If $A(x)=F_{x}^{\prime}, G(x)=0$ for each $x \in D \subseteq X$ and $L_{0}(t) \leq L(t)$ for each $t \in[0, r]$, then Theorem 1 reduces to [10, Theorem 2.2].

(4) For the choice of $G=0$, Corollary 2 reduces to the well known Newton Kantorovich theorem which was already discussed by Wang [11] and Tapia [12].

\section{Conclusions}

In this paper we improved and extend the inexact Newton-like methods $[7,8,10]$ in the context of differentiability of involved operator and introduced an inexact Newton-like algorithm for solving the generalized operator equations. We discussed the semilocal convergence analysis of our algorithm under the weak Lipschitz condition.

\section{References}

[1] Kantorovich, L.V. (1948). On Newton's method for functional equations. Doklady Akad. Nauk SSSR (N.S.), 59, 1237-1240 (Russian).

[2] Kantorovich, L.V., \& Akilov, G.P. (1982). Functional Analysis, Oxford: Pergamon Press.

[3] Argyros, I.K., Khattri, S.K., \& Hilout, S. (2013). Expanding the applicability of Inexact Newton Methods under Smale's $(\alpha, \gamma)$-theory. Appl. Maths. Comput., 224, 24-237.

[4] Morini, B. (1999). Convergence behaviour of inexact Newton methods. Math. Comput., 68, $1605-1613$

[5] Li, C., \& Shen, W.P. (2008). Local convergence of inexact methods under the Hölder condition. J. Comput. Appl. Math., 222, 544-560.

[6] Shen, W.P., \& Li, C. (2009). Kantorovich-type convergence criterion for inexact Newton methods. Appl. Numer. Math., 59, 1599-1611.

[7] Shen, W.P., \& Li, C. (2010). Smale's $\alpha$-theory for inexact Newton methods under the $\gamma$-condition. J. Math. Anal. Appl., 369, 29-42.

[8] Xu, X., Xiao, Y., \& Liu, T. (2012). Semilocal convergence analysis for inexact Newton method under weak condition. Abstract Appl. Anal., art. ID 982925, 13 p.

[9] Dembo, R.S., Eisenstat, S.C., \& Steihaug, T. (1982). Inexact Newton methods. Siam J. Numer. Anal., 19, 400-408.

[10] Argyros, I.K., \& Santosh, G. (2017). Exposnding the applicability of inexact Newton methods using restricted Convergence domain. Applicationes Mathematicae, 44(1), 123-133.

[11] Wang, X.H. (1999). Convergence of Newton's method and inverse function theorem in Banach space. Math. Comput., 68, 169-186.

[12] Tapia, R.A. (1971). The Kantorovich theorem for Newton's method. Amer. Math. Mon., 78, 389-392. 